\title{
Application of mixed models of random fields for the segmentation of satellite images
}

\author{
N A Andriyanov ${ }^{1}$ and V E Dement'ev ${ }^{1}$ \\ ${ }^{1}$ Ulyanovsk State Technical University, Severny Venets str. 32, Ulyanovsk, Russia, 432027
}

\begin{abstract}
The problem of images segmentation is considered in the article. A brief overview of the existing segmentation methods is provided. We suggested to use estimates obtained in the course of nonlinear recurrent filtering for segmentation of inhomogeneous images. The proposed segmentation algorithm was investigated when working with generated images and real ones. It is shown that effective estimation of model parameters can provide the best quality of segmentation in comparison with the ISODATA algorithm. In addition, it is shown how it is possible to modify the segmentation model used to find the boundaries between objects.
\end{abstract}

\section{Introduction}

Recently, the problems associated with the development and research of image processing algorithms and video sequences in various machine vision systems have become especially topical. This is due to the constantly increasing volume of stored and processed digital images and the growth of the capabilities of modern computer technology. A typical example of such systems is a variety of space complexes that provide data for remote sensing of the Earth (RS). Satellite imagery is widely used for monitoring the state of the atmosphere, the surface of the oceans, polar territories, agricultural lands, urban areas, deserts and forests.

An important obstacle to the wide use of high-resolution data is the limitations in the tools used, which provide an automated analysis and interpretation of such data. One of the fundamental stages in the processing of images is their segmentation, which is carried out to divide the image into segments containing pixels similar in their visual characteristics. Each pixel is assigned a certain label (the number of the segment to which it is assigned), followed by the formation of a segment map. Such processing allows, for example, to single out on a satellite image homogeneous areas (forest, field, urban development, etc.), the subsequent analysis of which is much simpler in comparison with the study of the original heterogeneous satellite image.

\section{Basic groups of segmentation algorithms}

Most existing and used image segmentation techniques can be divided into the following main groups [1].

1. First group is segmentation algorithms based on visual homogeneity of the area. The methods of this group use the homogeneity criterion for obtaining connected image areas $[1,2,7,8]$.

2. Second group includes segmentation algorithms based on the delineation of boundaries $[2,3,6,10]$. The methods of this group are based on the hypothesis of the discontinuity of the brightness properties of the image during the transition from one homogeneous region to another, i.e. on the 
existence of the edges of regions. The edges belong to the boundaries of the regions corresponding to the segmentation result. Methods for selecting edges are divided into local and global.

3 . The third group is segmentation methods based on histogram analysis $[1,2,4,11]$. These methods use the construction of one or more histograms for a given color image, finding histogram peaks, determining the intervals containing these peaks, and using these intervals to classify the pixels.

4. The fourth group may be considered as fuzzy segmentation. Fuzzy clustering in color or multispectral space [4,5] using, among other things, neural network solutions. The methods of this group are focused on finding the preliminary number and characteristics of homogeneous regions, for example, by fuzzy analysis of one-dimensional histograms calculated for each base color. This analysis allows you to detect peaks of histograms and at the same time determines the intervals around these peaks.

5. Segmentation algorithms based on physical properties of the image $[1,2,9]$ is the main component of fifth group. A special feature of the methods of this group is the orientation to the selection of the area corresponding to the image of the real physical object.

\section{Estimation of parameters and segmentation of simulated images}

In this paper, we investigate the possibility of using not the brightness values of individual pixels, but the correlation characteristics of these images for image segmentation. To obtain such correlation characteristics, let us use the description of a segmented image using a doubly stochastic model [12$16]$.

So the image is considered as random field (RF) given on a rectangular multidimensional grid $\Omega$. The method of such a representation implies that its values $x_{\bar{i}}=F\left(x_{\bar{j}}, \alpha_{\bar{i}}, \xi_{i}\right)$, where $\bar{i}, \bar{j} \in \Omega, \bar{j} \in D_{\bar{i}}$; $D_{\bar{i}} \in \Omega$ is model definition domain at $\bar{i} ; \quad F()$ is some transformation; $\alpha_{\bar{i}}$ are model parameters, which are RF independent of $\xi_{\bar{i}}$. A simple example of a doubly stochastic model is the following construction defined on a two-dimensional grid $\Omega=\left\{i=1,2, . . M_{1} ; j=1,2, . . M_{2}\right\}$. Such a model uses a combination of autoregressive models with multiple roots of the characteristic equations of multiplicity 2 and 1 [17]. Thus, we can write the particular doubly stochastic model as following

$$
\begin{aligned}
& x_{i j}=2 \rho_{x i j} x_{i-1, j}+2 \rho_{y i j} x_{i, j-1}-4 \rho_{x i j} \rho_{y i j} x_{i-1, j-1}-\rho_{x i j}^{2} x_{i-2, j}-\rho_{y i j}^{2} x_{i, j-2}+ \\
& +2 \rho_{x i j}^{2} \rho_{y i j} x_{i-2, j-1}+2 \rho_{y i j}^{2} \rho_{x i j} x_{i-1, j-2}-\rho_{x i j}^{2} \rho_{y i j}^{2} x_{i-2, j-2}+\xi_{i j},
\end{aligned}
$$

where $\xi_{i j}$ is independent random variable (RV) with Gaussian distribution; $M\left(\xi_{i j}\right)=0 ; M\left(\xi_{i j}{ }^{2}\right)=\sigma_{\xi}^{2}$, and $\left\{\rho_{1 i j}, i=1,2, \ldots, M_{1}, j=1,2, \ldots, M_{2}\right\}$ and $\left\{\rho_{2 i j}, i=1,2, \ldots, M_{1}, j=1,2, \ldots, M_{2}\right\}$ are a set of correlation parameters that obey the following relations:

$$
\begin{aligned}
& \rho_{1 i j}=r_{11} \rho_{1(i-1) j}+r_{12} \rho_{1 i(j-1)}-r_{11} r_{12} \rho_{1(i-1)(j-1)}+\xi_{1 i j}, \\
& \rho_{2 i j}=r_{21} \rho_{2(i-1) j}+r_{22} \rho_{2 i(j-1)}-r_{21} r_{22} \rho_{2(i-1)(j-1)}+\xi_{2 i j},
\end{aligned}
$$

where $\left\{\xi_{1 i j}\right\}$ and $\left\{\xi_{2 i j}\right\}$ are two-dimensional RFs of independent Gaussian RV with zero means and variances $M\left\{\xi_{1 j}^{2}\right\}=\left(1-r_{11}^{2}\right)\left(1-r_{12}^{2}\right) \sigma_{\rho 1}^{2}, M\left\{\xi_{2 i j}^{2}\right\}=\left(1-r_{21}^{2}\right)\left(1-r_{22}^{2}\right) \sigma_{\rho 2}^{2} ; \sigma_{\rho 1}^{2}=M\left\{\rho_{1 i j}^{2}\right\}, \sigma_{\rho 2}^{2}=M\left\{\rho_{2 i j}^{2}\right\}$. For convenience, we can denote $r_{11}$ and $r_{12}$ as $r_{\mathrm{x} 1}$ and $r_{\mathrm{x} 2}$ respectively, and $r_{21}$ and $r_{22}$ as $r_{\mathrm{y} 1}$ and $\mathrm{r}_{\mathrm{y} 2}$.

It should be noted that for similar autoregressive doubly stochastic models, it is possible to construct recurrent nonlinear filtration procedures that allow the estimation of both brightness properties $\left(\left\{x_{i j}: i=1,2, . . M_{1} ; j=1,2, . . M_{2}\right\}\right)$ and correlation properties $\left(\left\{\rho_{1 i j}, \rho_{2 i j}: i=1,2, . . M_{1} ; j=1,2, . . M_{2}\right\}\right)$.

For this we compose the following vector length $4 M_{1}+5$ elements:

$$
\begin{aligned}
& \bar{x}_{i j}=\left(\begin{array}{lllllllllll}
\bar{x}_{x i j} & \bar{\rho}_{x i j} & \bar{\rho}_{y i j}
\end{array}\right)^{T}, \\
& \bar{x}_{x i j}=\left(\begin{array}{llllllllllll}
x_{i-1 M_{1}} x_{i j} & x_{i j-1} & \ldots & x_{i 1} & x_{i-1 M_{1}} & \ldots & x_{i-11} & x_{i-2 M} & \ldots & x_{i-2 j-2}
\end{array}\right)^{T}, \\
& \bar{\rho}_{x i j}=\left(\begin{array}{lllllllllll}
\rho_{x i j} & \rho_{x i j-1} & \ldots & \rho_{x i 1} & \rho_{x i-1 M_{1}} & \ldots & \rho_{x i-1 j}
\end{array}\right)^{T}, \bar{\rho}_{y i j}=\left(\begin{array}{llllllll}
\rho_{y i j} & \rho_{y i j-1} & \ldots & \rho_{y i 1} & \rho_{y i-1 M_{1}} & \ldots & \rho_{y i-1 j}
\end{array}\right)^{T} .
\end{aligned}
$$

Then the RF model will be written in the form 


$$
\bar{x}_{i j}=\wp_{i j} \bar{x}_{i j-1}+\bar{\xi}_{i j}
$$

where $\wp_{i j}=\left(\begin{array}{ccc}\wp_{i j x} & 0 & 0 \\ 0 & \wp_{i j \rho x} & 0 \\ 0 & 0 & \wp_{i j \rho y}\end{array}\right)$ is matrix having size $\left(4 M_{1}+5\right) \times\left(4 M_{1}+5\right)$.

The first row of the matrix $\wp_{i j x}$ is equal to

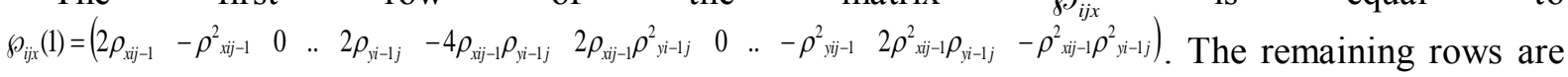
composed by attaching a zero column to the identity matrix.

$$
\begin{aligned}
& \wp_{i j x}=\left(\begin{array}{cccccccccccc}
2 \rho_{x i j-1} & -\rho_{x i j-1}^{2} & 0 & . . & 2 \rho_{y i-1 j} & -4 \rho_{x i j-1} \rho_{y i-1 j} & 2 \rho_{x i j-1} \rho_{y i-1 j}^{2} & 0 & . . & -\rho_{y j-1}^{2} & 2 \rho_{x i j-1}^{2} \rho_{y i-1 j} & -\rho_{x i j-1}^{2} \rho_{y i-1 j}^{2} \\
1 & 0 & 0 & . . & 0 & 0 & 0 & 0 & . . & 0 & 0 & 0 \\
0 & 1 & 0 & . . & 0 & 0 & 0 & 0 & . . & 0 & 0 & 0 \\
0 & 0 & 1 & . . & 0 & 0 & 0 & 0 & . . & 0 & 0 & 0 \\
0 & 0 & 0 & . . & 0 & 0 & 0 & 0 & . . & 0 & 0 & 0 \\
0 & 0 & 0 & . . & 1 & 0 & 0 & 0 & . . & 0 & 0 & 0 \\
0 & 0 & 0 & . . & 0 & 1 & 0 & 0 & . . & 0 & 0 & 0 \\
0 & 0 & 0 & . . & 0 & 0 & 1 & 0 & . . & 0 & 0 & 0 \\
0 & 0 & 0 & . . & 0 & 0 & 0 & 1 & . . & 0 & 0 & 0 \\
0 & 0 & 0 & . . & 0 & 0 & 0 & 0 & . . & 0 & 0 & 0 \\
0 & 0 & 0 & . . & 0 & 0 & 0 & 0 & . . & 1 & 0 & 0 \\
0 & 0 & 0 & . . & 0 & 0 & 0 & 0 & . . & 0 & 1 & 0
\end{array}\right) \\
& \wp_{i j \rho x}=\left(\begin{array}{cccc}
r_{x 1} & \ldots & r_{x 2} & -r_{x 1} r_{x 2} \\
1 & \ldots & 0 & 0 \\
\ldots & \ldots & \ldots & \ldots \\
0 & \ldots & 1 & 0
\end{array}\right) ; \wp_{i j \rho y}=\left(\begin{array}{cccc}
r_{y 1} & \ldots & r_{y 2} & -r_{y 1} r_{y 2} \\
1 & \ldots & 0 & 0 \\
\ldots & \ldots & \ldots & \ldots \\
0 & \ldots & 1 & 0
\end{array}\right) .
\end{aligned}
$$

We represent these matrix relations in the form of the following formula $\bar{x}_{i j}=\varphi\left(\bar{x}_{i j-1}\right)+\bar{\xi}_{i j}$. We introduce the extrapolated estimate $\hat{\bar{x}}_{i j}=\varphi\left(\bar{x}_{i j-1}\right)$ and we find the matrix $\varphi^{\prime}\left(\bar{x}_{i j-1}\right)=\frac{\partial \varphi\left(\bar{x}_{i j-1}\right)}{\partial \bar{x}_{i j-1}}$. Direct calculations show that it will be identical to the matrix $\wp_{i j}$, except for the first line, which will be equal to $\varphi_{1}^{\prime}=\left(\begin{array}{lll}A_{1} & A_{2} & A_{3}\end{array}\right)$, where

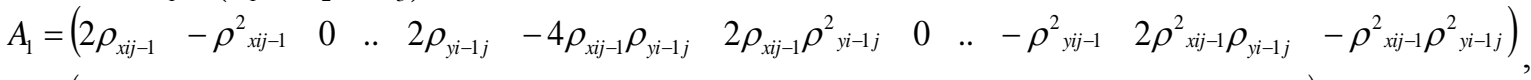

$$
\begin{aligned}
& A_{2}=\left(\begin{array}{lllll}
2 x_{i-1 j}-4 \rho_{y i-1 j} x_{i-1 j-1}-2 \rho_{x i j-1} x_{i-2, j}+4 \rho_{x i j-1} \rho_{y i-1 j} x_{i-2, j-1}+2 \rho^{2}{ }_{y i-1 j} x_{i-1, j-2}-2 \rho_{x i j-1} \rho_{y i-1 j}^{2} x_{i-2, j-2} & \ldots & 0 & 0
\end{array}\right) \text {, } \\
& A_{3}=\left(\begin{array}{llll}
0 & \ldots & 2 x_{i j-1}-4 \rho_{x i j-1} x_{i-1 j-1}-2 \rho_{x i j-1} x_{i, j-2}+4 \rho_{x i j-1} \rho_{y i-1 j} x_{i-1, j-2}+2 \rho_{x i j-1}^{2} x_{i-2, j-1}-2 \rho_{x i j-1}^{2} \rho_{y i-1 j} x_{i-2, j-2} \quad 0
\end{array}\right) .
\end{aligned}
$$

$A_{1}, A_{2}, A_{3}$ are rows consisting of $M_{1}+1$ elements.

Using these relations and the method of recurrent vector filtration, we can write the following twodimensional nonlinear filter:

$$
\hat{\bar{x}}_{i j}=\hat{\bar{x}}_{3 i j}+B_{i j}\left(z_{i j}-\hat{x}_{i j}\right),
$$

where $\hat{x}_{3 i j}$ is the first element of the vector $\hat{\bar{x}}_{i j} ; B_{i j}=P_{i j} C^{T} D_{i j}^{-1} ; C=(1,0,0, \ldots, 0) ; D_{i j}=C P_{i j} C^{T}+\sigma_{n}^{2}$.

It is important that the estimated brightness and correlation characteristics are constructed as a result of a consistent evaluation of the entire image. This allows for a higher quality of evaluation than, for example, in a sliding window.

Arrays of estimates $\left\{\rho_{1 i j}, \rho_{2 i j}: i=1,2, . M_{1} ; j=1,2, . M_{2}\right\}$ obtained during nonlinear filtering can be considered as two-dimensional arrays characterizing the correlation properties of the original image. 
Accordingly, various processing algorithms can be applied to them, including the segmentation procedures.

Figure 1(a) shows simulated image obtained using the model (2). In this image there are two types of objects, close in brightness characteristics, but differing in correlation properties. Figure 1(b) shows field of auxiliary correlation parameters for the original image after filtering (3), and Figure 1(c) shows histogram for these correlation parameters. It shows two characteristic peaks separated by a local extremum. Using this extremum as a boundary, it is possible to perform a simple partition of the correlation parameter field and the original image into two disjoint regions. Figure 1(d) shows the results of such segmentation.

(a)

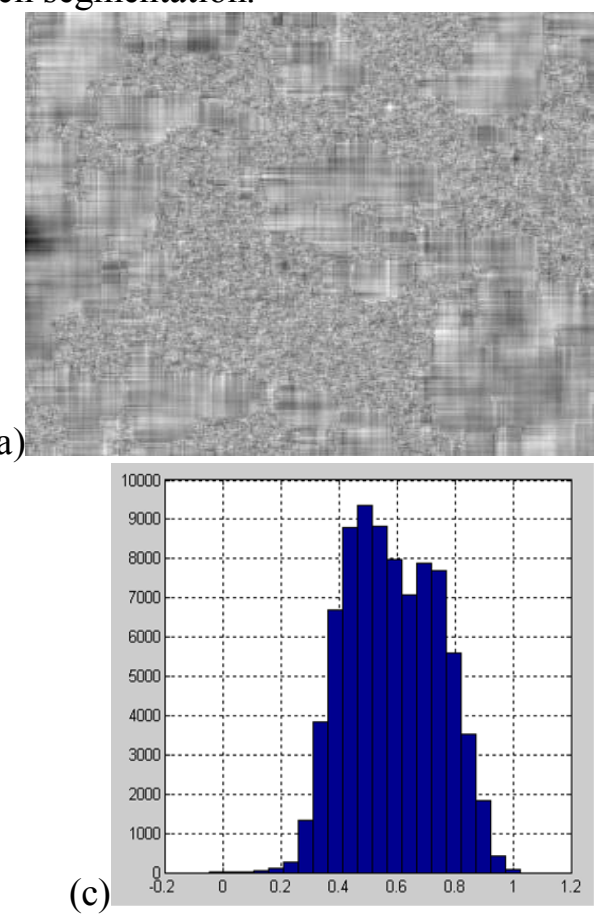

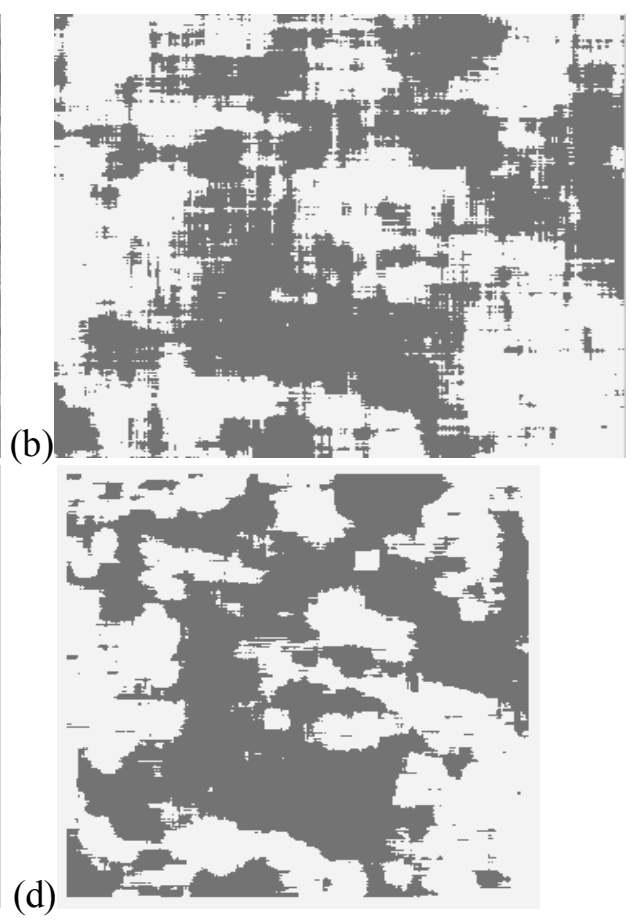

(d)

Figure 1. Segmentation of an image with varying correlation properties.

The analysis of the obtained results testifies to the high quality of the segmentation performed. About $89 \%$ of the original image points were segmented correctly. However, it should be noted that the peaks of the histogram of the correlation parameters in this case corresponded to the values of the correlation coefficients 0.5 and 0.7 , respectively, i.e. which differ by approximately $27 \%$.

Thus, the main advantage of the proposed model in comparison with the known ones is the possibility of taking into account the internal connections between the pixels during segmentation, in addition to methods based only on the brightness of specific pixels. However, the use of preprocessing requires more computational complexity than the simple application of known algorithms, such as ISODATA, k-means, MRF-segmentation.

Table 1 shows the time taken for image segmentation performed on a PC AMD-FX 4350 QuadCore $4.2 \mathrm{GHz}, 8 \mathrm{~Gb}$ RAM. The image size is $300 \times 300$.

Table 1. The complexity of segmentation.

\begin{tabular}{ccc}
\hline № & Algorithm & Time, sec \\
\hline 1 & k-means & 0,5 \\
2 & ISODATA & 0,78 \\
3 & Nonlinear filtering + k-means & 11,2 \\
4 & Nonlinear filtering + ISODATA & 11,5 \\
\hline
\end{tabular}

Analysis of the data in Table 1 shows that the proposed algorithm reduces the processing speed, but in some cases it allows to achieve an acceptable segmentation in comparison with the known methods. Increase the speed can be using window estimation of parameters, instead of a nonlinear filter. 


\section{Results of segmentation of real images}

Nevertheless, the result obtained for simulated images allows us to reasonably hope that in the case of applying more complex segmentation procedures in processing the correlation parameter field, the segmentation algorithm found can be applied to real images.

Indeed, Figures 2-7 presents the results of segmentation (binarization) of some typical images by applying a combination of the proposed algorithm and the ISODATA algorithm applied to the correlation parameter field. Figures 2(a)-7(a) show the original images, Figures 2(b)-7(b) show corresponding fields of correlation parameters, Figures 2(c)-7(c) shows results of segmentation based on correlation properties, Figures 2(d)-7(d) show segmentation results with intermediate subsampling. It means, that the estimate of the parameters was averaged over some small neighborhood. Finally, Figures 2(e)-7(e) shows the results of applying the ISODATA algorithm to the original images.

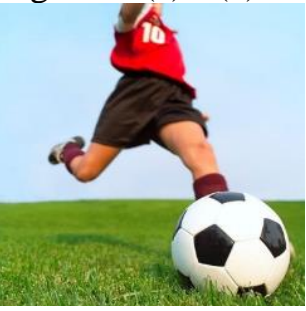

(a)

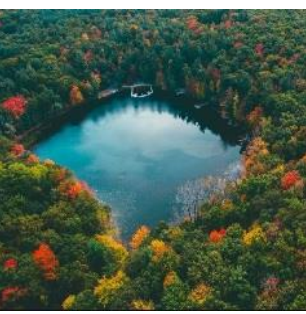

(a)

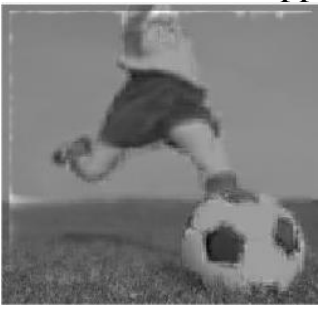

(b)

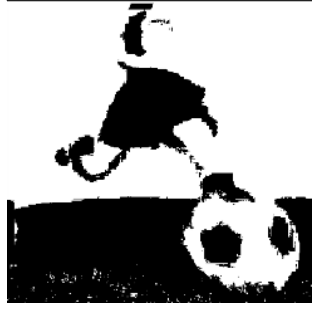

(c)

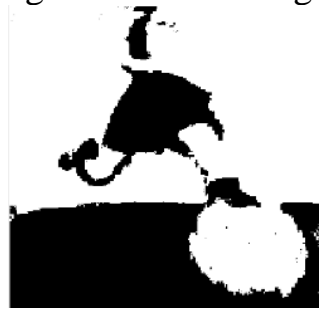

(d)

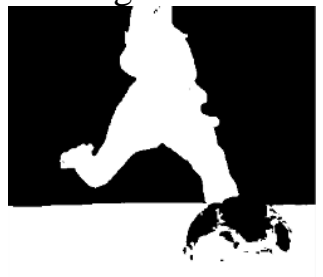

(e)

Figure 2. Segmentation of a complex color image.

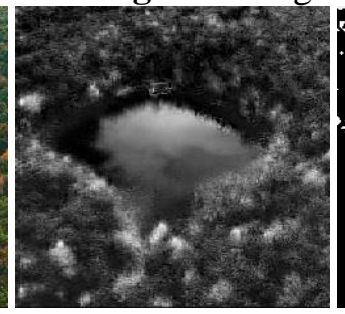

(b)

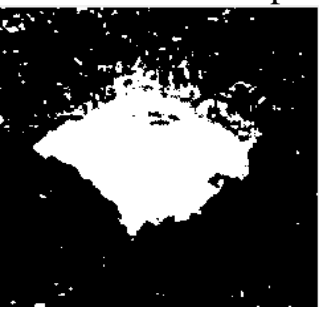

(c)

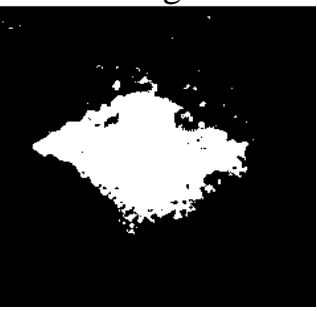

(d)

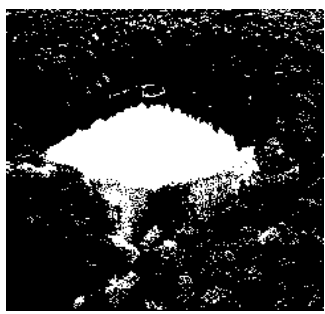

(e)

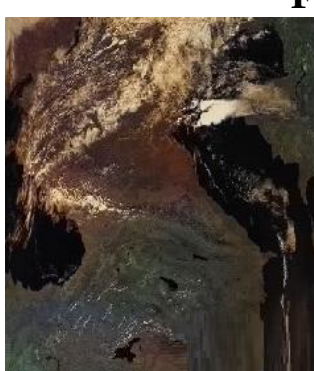

(a)

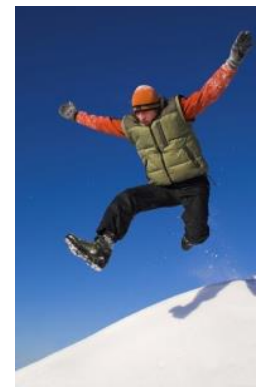

(a)

Figure 3. Segmentation of an image containing 2 distinct objects

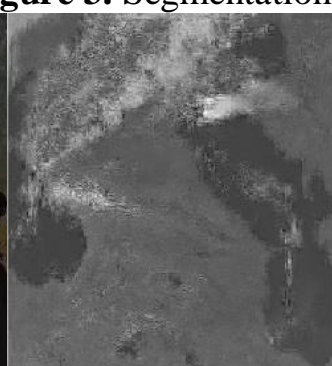

(b)

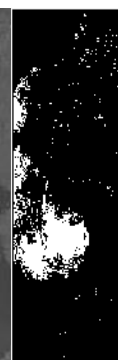

(c)

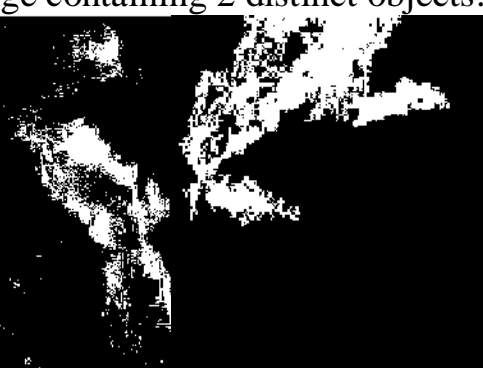

(d)

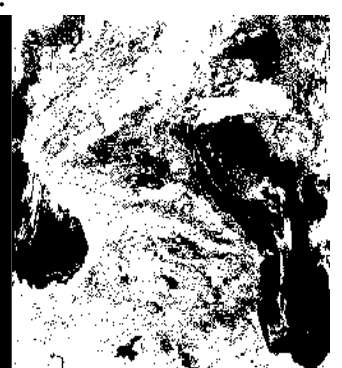

(e)

Figure 4. Segmentation of the satellite image (cloud-lake-ground).

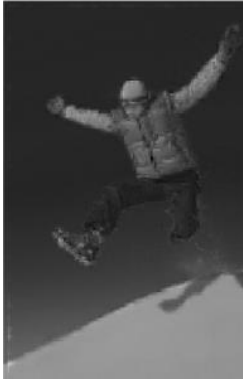

(b)

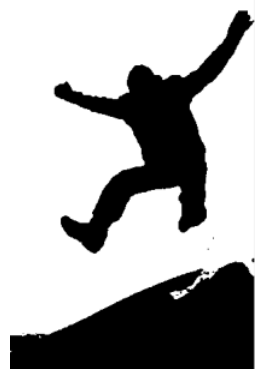

(c)

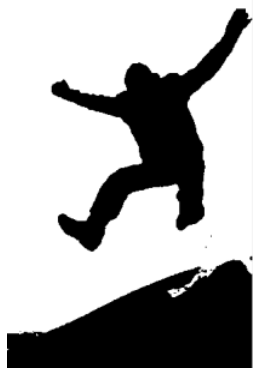

(d)

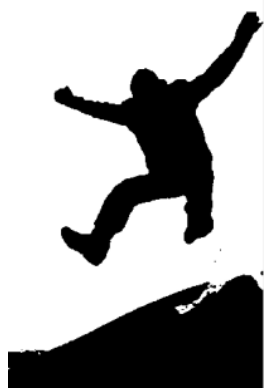

(e)

Figure 5. Segmentation of an image with pronounced luminance characteristics of objects. 


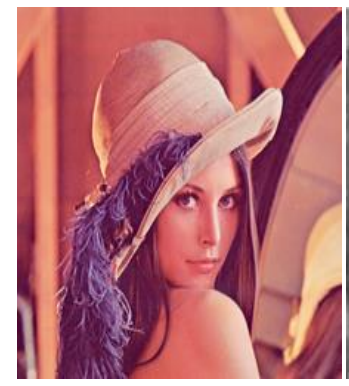

(a)

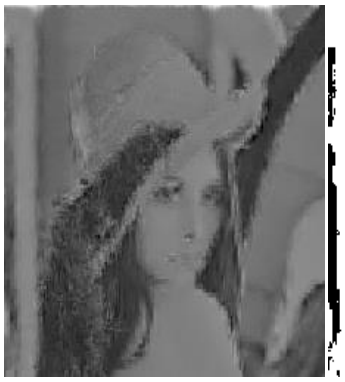

(b)

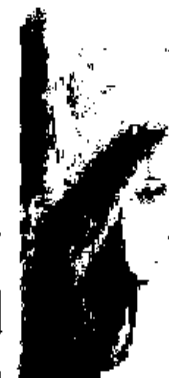

(c)

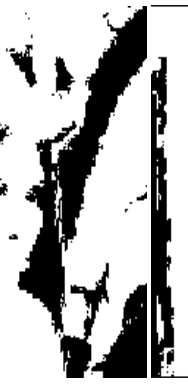

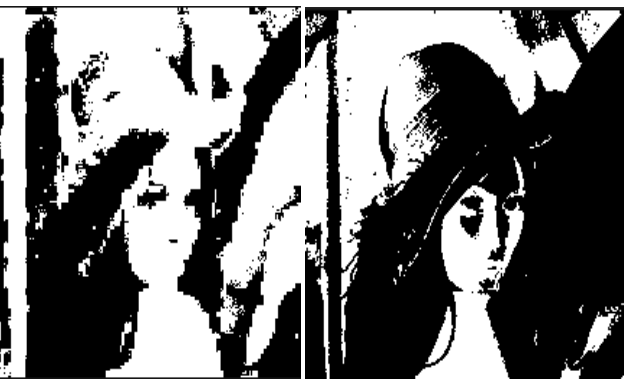

(d)

(e)

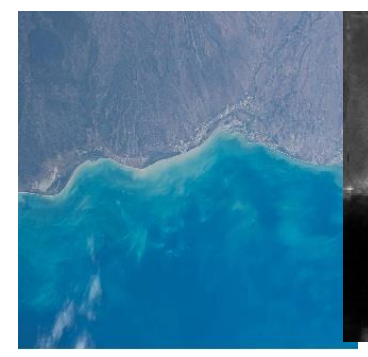

(a)

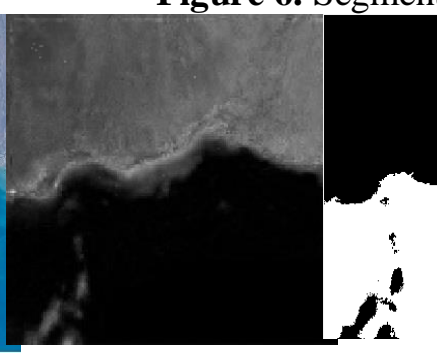

(b)

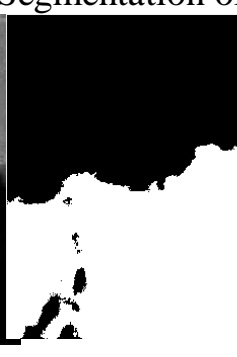

(c)

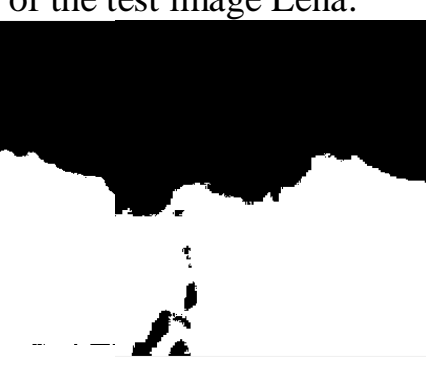

(d)

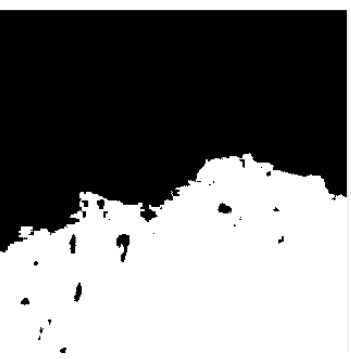

(e)

Figure 7. Segmentation of satellite image (ground-water).

The analysis of the given images, as well as the direct calculation of correctly assigned pixels, allows us to draw the following conclusions. First, the field of correlation parameters allows you to visually distinguish the objects existing on the source images. This gives base for using such a field for further processing, in particular segmentation. Secondly, in most cases (5 of 6) preliminary nonlinear filtering allowed to increase the quality of segmentation by an average of $8 \%$. In this case, the gain is greater, the more noticeable is the difference between the correlation properties of objects in comparison with the luminance ones.

Finally, Figures 8 and 9 show the results of automatic segmentation of image data and segmentation, taking into account the selection of 2 objects based on the MRF-segmentation algorithm.

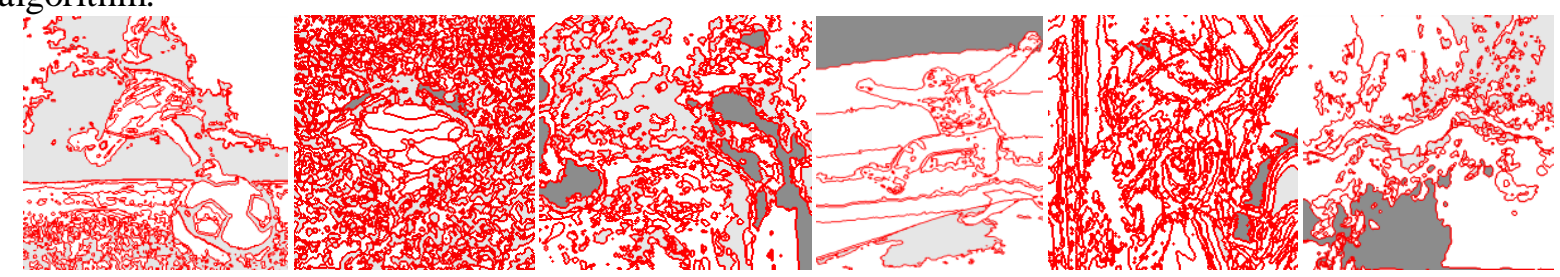

Figure 8. MRF-segmentation with auto-determination.
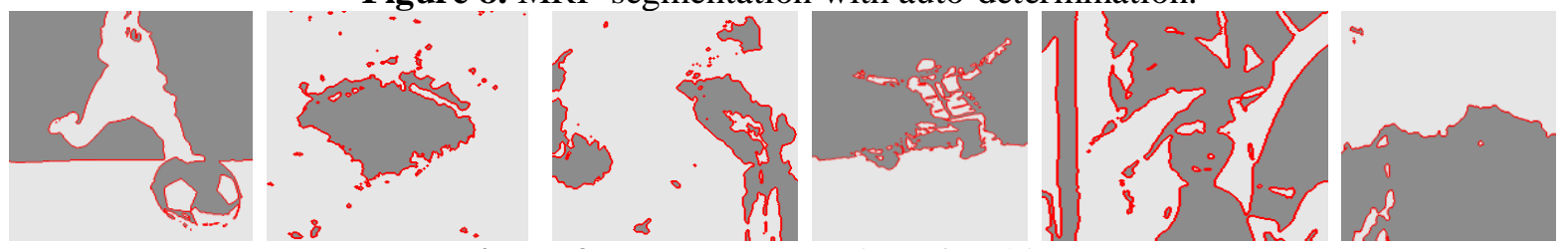

Figure 9. MRF-segmentation of 2 objects.

Obviously, such segmentation based on auto-determination of the number of objects is inefficient. When the algorithm indicates the number of objects of interest, then for some images, adequate segmentation is obtained, but an error remains about of $10-12 \%$ level. And in more complex images, segmentation remains unsatisfactory. Preliminary evaluation of the relationships between pixels helps either to eliminate errors in simple images, or to perform adequate segmentation (about 90\%) for complex images. 


\section{Boundaries detection}

Note that the use of the recurrent filter as a tool for obtaining and brightness and correlation characteristics of the image allows, among other things, to solve the problem of directly determining the boundaries between objects in images. To do this, in many cases it is sufficient to filter the image in the forward and backward directions $\left\{\overline{\hat{x}}_{\bar{l}}=\left(\hat{x}_{\bar{l}}, \hat{\alpha}_{i}, \hat{\rho}_{1 \bar{l}}, \ldots, \hat{\rho}_{N i}, \hat{\beta}_{\bar{l}}\right)\right\}$ and $\left\{\overline{\hat{\hat{x}}}_{\bar{l}}=\left(\hat{\hat{x}}_{\bar{i}}, \hat{\hat{\alpha}}_{\bar{l}}, \hat{\hat{\rho}}_{1 \bar{l}}, \ldots, \hat{\hat{\rho}}_{N i}, \hat{\hat{\beta}}_{\bar{l}}\right)\right\}$ Then it is necessary to determine statistics L for neighboring points $(\bar{i})$ and $(\bar{j})$. It can be calculated as

$$
L=\sum_{\bar{l} \in D \bar{i}} K_{1}(l)\left(\overline{\hat{x}}_{\bar{l}}-\overline{\hat{x}}_{\bar{l}}\right)^{T}+\sum_{\bar{l} \in D \bar{j}} K_{2}(l)\left(\overline{\hat{x}}_{\bar{l}}-\overline{\hat{x}}_{\bar{l}}\right)^{T},
$$

where $K_{1}(\bar{l})$ and $K_{2}(\bar{l})$ are vector coefficients of statistics L.

In the case where $\mathrm{L}$ is greater than the threshold value $\mathrm{L}_{0}$, it is decided that there is a boundary between the points $(\bar{i})$ and $(\bar{j})$. Based on the modified likelihood ratio, one can show the validity of this decision rule. The sense of the detector is related to the fact that the doubly stochastic filter, when passing the explicit boundary between two objects, each of which is described by its doubly stochastic model implementation, demonstrates a short-term increase in the variance of the estimation error. This increase is the more, then the difference between these objects is more obvious. You can detect this jump in the variance by comparing the estimates of the forward and backward filters.

Figure 10 shows artificial doubly stochastic image (see Figure 10(a)), calculated statistics L (see Figure 10(b)), fragment of a real satellite image (see Figure 10(c)) and the calculated statistics L for real image (see Figure 10(d)).
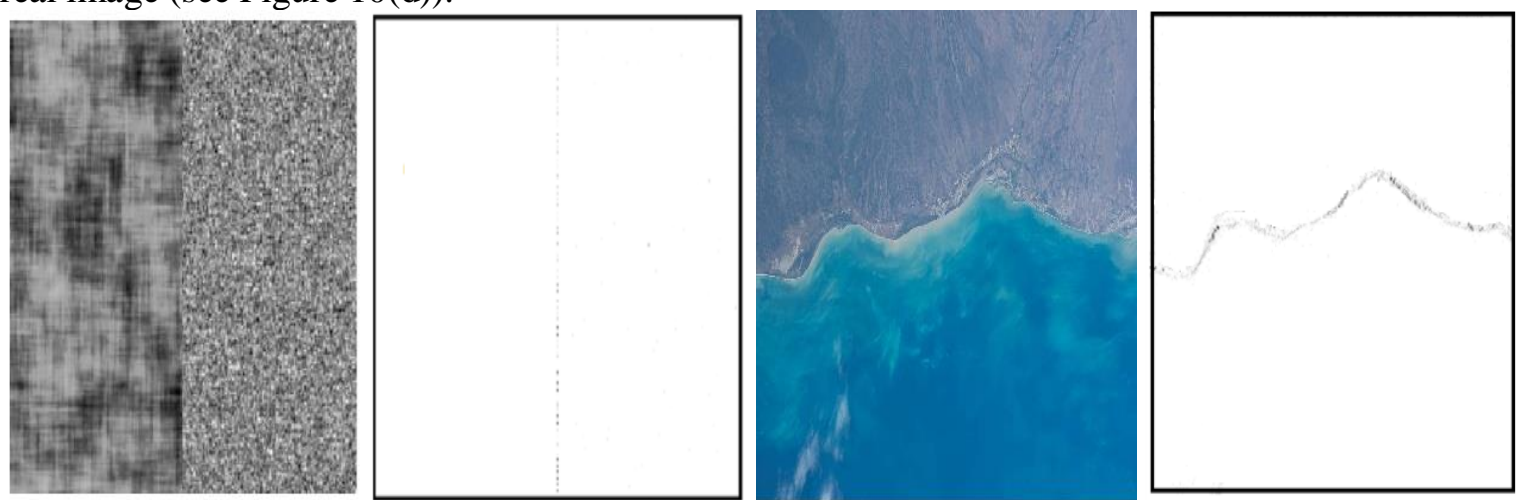

Figure 10. Selecting borders on an image using a two-pass doubly stochastic filter.

Thus, based on the proposed models, it is possible to detect boundaries between objects, which also makes it possible to improve the quality of segmentation.

\section{Conclusion}

Thus, the results of the conducted studies confirmed the possibility of using nonlinear recurrent filtering as an auxiliary tool that allows to improve the quality of segmentation of images of various types. This allows us to recommend this type of treatment for real machine vision systems.

\section{References}

[1] Gonzalez R and Woods R 2006 Digital image processing (Moscow: Technosphere) p 812

[2] Bakut P A and Kolmogorov G S 1987 Image Segmentation: Methods for delimiting the boundaries of regions Foreign Radio Electronics 10 16-23

[3] Malik J, Belongie S, Leung T and Shi J 2001 Contour and texture analysis for image segmentation International Journal of Computer Vision 43(1) 7-27 DOI: 10.1023/ A: 1011174803800

[4] Li S and Wu D O 2015 Modularity-based image segmentation IEEE Transactions on Circuits and Systems for Video Technology 25(4) 570-581 DOI: 10.1109/TCSVT.2014.2360028

[5] Long J, Shelhamer E and Darrell T 2015 Fully convolutional networks for semantic 
segmentation IEEE Conference on Computer Vision and Pattern Recognition 3431-3440 DOI: 10.1109/CVPR.2015.7298965

[6] Cigla C and Alatan A A 2010 Efficient graph-based image segmentation via speeded-up turbo pixels ICIP 3013-3016 DOI: 10.1109/ICIP.2010.5653963

[7] Fursov V A, Bibikov S A and Baida O A 2014 A thematic classification of hyperspectral images in terms of the conjugacy index Computer Optics 38(1) 154-158

[8] Zimichev E A, Kazansky N L and Serafimovich P G 2014 Spatial classification of hyperspectral images using the k-means ++ clustering method Computer Optics 38(2) 281-286

[9] Blokhinov Yu B, Gorbachev V A, Rakutin Yu O and Nikitin A D 2018 Development of an algorithm for the semantic segmentation of real-time aerial photographs Computer Optics 42(1) 141-148 DOI: 10.18287/2412-6179-2018-42-1-141-148

[10] Belim S V and Larionov S B 2016 Algorithm of image segmentation, based on the search for communities on graphs Computer Optics 40(6) 904-910

[11] Yeruslanov R V, Orekhova M N and Dubrovin V N 2015 Segmentation of images of organs of the retroperitoneal space using computed tomographic images based on the level function Computer Optics 39(4) 592-599

[12] Vasil'ev K K, Dement'ev V E and Andriyanov N A 2015 Doubly stochastic models of images Pattern Recognition and Image Analysis 25(1) 105-110

[13] Vasiliev K K, Dementiev V E and Andriyanov N A 2017 Filtration and restoration of satellite images using doubly stochastic random fields CEUR Workshop Proceedings 1814 10-20

[14] Azanov P, Danilov A and Andriyanov N 2017 Development of software system for analysis and optimization of taxi services efficiency by statistical modeling methods CEUR Workshop Proceedings 1904 232-238

[15] Vasiliev K K and Andriyanov N A 2017 Synthesis and analysis of doubly stochastic models of images CEUR Workshop Proceedings 2005 145-154

[16] Andriyanov N A and Gavrilina Yu N 2018 Image Models and Segmentation Algorithms Based on Discrete Doubly Stochastic Autoregressions with Multiple Roots of Characteristic Equations CEUR Workshop Proceedings 2076 19-29

\section{Acknowledgments}

The study was supported by RFBR, project № 18-31-00056. 